Humberd, B.K., Clair, J.A., \& Creary, S.J. 2015. In our own backyard: When a less inclusive community challenges organizational inclusion. Equality, Diversity and Inclusion. 34(5): 395421.

\title{
IN OUR OWN BACKYARD: WHEN A LESS INCLUSIVE COMMUNITY CHALLENGES ORGANIZATIONAL INCLUSION
}

\begin{abstract}
Purpose: This paper aims to build insight into how the local community impacts an organization's ability to develop an inclusive culture. The paper offers a framework of inclusion discontinuities between an organization and its community, then using the case of teaching hospitals, aims to empirically demonstrate how individuals and organizations perceive and deal with inconsistencies in responses to diversity at the organizational-and community-levels.

Design/methodology/approach: A multi-method qualitative study was conducted in hospitals located in the same city. Focus groups were conducted with 11 medical trainees from underrepresented backgrounds and semi-structured interviews were conducted with 10 hospital leaders involved with diversity efforts at two hospitals. Data analysis followed an iterative approach built from Miles and Huberman (1994).

Findings: The findings demonstrate how discontinuity between the organizations efforts to create an inclusive environment and the community's response to diversity and difference creates challenges for leaders in retaining and supporting minority individuals, and for trainees in feeling like they could build a life within, and outside of, their organizations. Based on findings from the data, the paper offers insights into how organizations can build their capacity to address these challenges.
\end{abstract}

Research limitations/implications: Future research should build upon this work by further examining how inclusion discontinuities between communities and organizations impact individuals and organizations.

Practical implications: The paper includes in-depth insight into how organizations can build their capacity to address such a deep-rooted challenge that comes from a less inclusive community.

Originality/value: This paper contributes to an understanding of how forces from the community outside an organization can shape internal efforts toward fostering inclusion.

Keywords: organizational inclusion, community, organizational effectiveness 
With increasing demographic diversity in the United States and around the world, scholars and organizations alike are interested in understanding how organizations can foster inclusive cultures. Inclusive organizational cultures are those in which employees from different backgrounds can individually and collectively contribute and reach their fullest potential (Pless \& Maak, 2004). While there has been some progress, organizations continue to face challenges in creating and maintaining inclusive cultures that positively engage difference (Davidson, 2011). As such, much of the diversity management literature continues to focus on uncovering internal processes and structures that can help individuals and organizations work more effectively across difference.

Over two decades of research findings demonstrate that a myriad of organizational factors influence an organization's capacity to develop an inclusive culture. Namely, organizational cultural assumptions and beliefs concerning diversity (e.g., Thomas, 1991; Pless \& Maak, 2004), shared understanding of what is meant by inclusion (e.g., Ely \& Thomas, 2001), engaged leaders (e.g., Ainscow \& Sandill, 2010; Nishii \& Mayer, 2009), and HR systems and processes that promote a climate of inclusion (e.g., Cox, 1994) are all necessary for creating inclusive organizations. However, very little work attends to the role that the context outside of the organization's walls plays in fostering inclusive organizations. This oversight is striking given that organizational theorists have long been concerned with the relationship between organizations and their environments (e.g., Barnard, 1938; Katz \& Kahn, 1978; Selznick, 1949), recognizing that organizations are deeply embedded in local social systems (Marquis, Lounsbury \& Greenwood, 2011) that shape, and are shaped by, an organization's behavior (Freeman, 1984; Mitroff, 1983). Like organizations, these social systems have their own cultural assumptions and beliefs concerning diversity, demographic patterns of integration and segregation, and an 
institutional environment that can privilege or disadvantage individuals. Thus, as Brief et al (2005: 839) stated: "It is time that researchers concerned with relationships among demographically different groups in organizations look outside the organizations they study to better understand what is happening within them.”

In this paper, we propose that greater attention to the role of the local community is critical to advancing research and praxis on diversity and inclusion in organizations. The research we report here is part of a larger inductive investigation interested in understanding how racially and ethnically underrepresented medical residents (heretofore referred to as "trainees") who are new to a profession experience challenges and opportunities related to their underrepresented (minority) status in their professions and hospital organizations. Through this investigation, it became apparent to us that while trainees perceived their residency programs and teaching hospitals to be committed to inclusion, they did not perceive the same degree of commitment from the local community. The latter became a barrier to each hospital's ability to develop an inclusive culture. Thus, in this paper, we build insight into how the local community impacts an organization's ability to develop an inclusive culture and the ways in which an organization can proactively address this tension. Using the case of teaching hospitals, we draw on in-depth qualitative data to consider how organizational leaders and trainees perceive inconsistencies in responses to diversity at the organizational- and community-levels. We then offer insights into how organizations can address these challenges based on the findings from our data. In so doing, we address the following research question: How can organizations foster an inclusive environment within, when the local community in which those organizations is situated lacks that same focus? 
The results of our study emphasize how vital it is for organizations to recognize that inconsistencies may exist between the nature of inclusion within an organization and within its community, which we refer to as "inclusion discontinuities." Our assertion here is that inclusion discontinuities have an often silent, but critical effect on key performance measures for a diverse organization, such as the organization's ability to recruit and retain a diverse workforce.

Therefore, in this paper, we conceptualize inclusion discontinuity and empirically illustrate how organizations focused on building an inclusive culture dealt with challenges arising from their location within a community that was perceived to be less inclusive. We demonstrate that inclusion discontinuities shape how the organization can promote an experience of inclusion, particularly for those employees who come from historically underrepresented or marginalized social identity groups.

Our work fits within an emerging trend in diversity scholarship in which scholars have responded to the call to more deeply engage the external context of organizations in diversity research (e.g., Avery, McKay \& Wilson, 2008, Brief et al, 2005; Joshi \& Roh, 2009; King et al, 2011; Pugh et al, 2008; Ragins et al, 2012). Though only a handful of studies have thus far been reported, existing work reveals important relationships between community-related variables and job-relevant outcomes for individuals. Our work extends these studies by building insight into the role that difference between an organization's inclusiveness and a community's inclusiveness plays in an organization's ability to create and maintain an inclusive environment. Through our study, we demonstrate that organizations cannot merely focus internally on building an inclusive culture; they must also consider how the community responds to diversity, particularly, when those responses are not aligned with the organization's vision for inclusion. 
To explore these connections, first, we develop the concept of inclusion discontinuities by articulating the various ways in which an organization and its local community may be inconsistent in terms of diversity. Then, we draw on data from our research to empirically illustrate how embeddedness in a community that is less inclusive can often work against an organization's efforts. Through our analysis, we illustrate how organizational leaders and individual employees experience the challenges created by this discontinuity with the community, and we offer suggestions for how an organization may engage the community to work more effectively across differences.

\section{A FRAMEWORK OF INCLUSION DISCONTINUITIES}

A small body of work has begun to explore how the community outside of an organization may influence diversity-relevant outcomes within the organization. Below, we review and build from this work to offer a framework for forms of inclusion discontinuities.

\section{Defining Inclusion Discontinuity}

Discontinuity is defined as “... a quality or state of not being continuous," as a break or an inconsistency between one thing and another (Merriam-Webster, 2013). Discontinuities are common across a wide array of human experience, some occurring specifically within organizations - ranging from discontinuities in group development processes as described in the classic punctuated equilibrium model of team progression (Gersick, 1988) to discontinuities in the quality of work processes across different functional areas in an organization (e.g., discontinuities of care across in-patient and out-patient services in a hospital) (e.g., Moore, Wisnivesky, Williams, \& McGinn, 2003). In this paper, we additionally highlight that discontinuities can also include breaks or inconsistencies between the nature of an organization and the nature of an organization's local community. 
Specifically, we define "inclusion discontinuities" as inconsistencies between inclusiveness within the boundaries of the organization and inclusiveness within the local community in which the organization operates. While it is possible for the local community to be more inclusive than an organization, in this paper, we focus on instances in which an organization is more inclusive than the local community. In such a case, employees may feel included at work, but they may experience greater marginalization and sense of injustice when they leave the organization. Such discontinuity creates a complex and deep-rooted challenge for organizations seeking to foster an inclusive culture.

\section{Forms of Inclusion Discontinuity}

Inclusion discontinuities can take several forms, as laid out in Table 1. Importantly, these forms are not mutually exclusive or exhaustive, but rather are likely to be simultaneous processes with recursive influence on one another. We consider each in turn.

First, inclusion discontinuity can take the form of an inconsistency between demographic representation within the organization and the demographic representation in the embedded community. Much of the existing diversity work that has considered community context tends to reflect a concern with this form of inclusion discontinuity. For instance, some research considers how demographic matching between employees within an organization and customers (within the community) may have important implications for organizational performance (e.g., Leonard, Levine \& Joshi, 2004; Sacco \& Schmitt, 2005). Much of this work examines assumptions associated with the "access and legitimacy" paradigm of diversity management (Ely \& Thomas, 2001), which suggests that the greater the match of an organization's demographic composition to the demographics of the community, the more likely an organization will be able to target a 
diverse customer base. For instance, an organization may be better able to garner insight into the concerns or interests of the Hispanic community if they employ Hispanic individuals.

Other research considers the influence of community demographic variables on diversityrelevant outcomes in organizations. For example, studies examine how racial composition of a community impacts workplace discrimination reports (Avery et al, 2008), perceptions of an organization's diversity climate (Pugh et al, 2008), and job acceptance decisions of minority and majority group applicants (McKay \& Avery, 2006). King et al (2011) focus more specifically on the inconsistency by calculating a representation ratio of ethnic diversity within the organization relative to the community. They find that this ratio of representation matters for the level of incivility displayed in organizations.

The second form of inclusion discontinuity concerns inconsistencies between the power and status afforded to employees based on their social identities such as race or gender within the organization compared to the power and status afforded to these same employees within their local community. As one illustration, a Black professional may work in an organization in which Black employees are relatively well represented in higher-status and well-paid professional roles. However, in wealthy areas in the local community, there may be few Black professionals represented. This form of inclusion discontinuity recognizes the role of social stratification, that when representation coalesces around particular levels of economic or professional hierarchy it creates systematic difference in the power and status of particular social groups (Lenski, 1966; Gordon, Edwards \& Reich, 1986). Recent work echoes such a focus; for instance, in their conceptual paper, McKay \& Avery (2006: 408) suggest that community diversity vertical integration, (which refers to "the perceived proportional representation of a given racioethnic group in the firm's community, across various social classes"), is an important influence on 
minority and majority group applicants' job acceptance intentions. In an unpublished dissertation, Garnett (2012) examines how differences in how minorities and women are segregated across occupational categories in the local communities in which firms are embedded have implications for workplace inequality and discrimination.

The third type of inclusion discontinuity involves inconsistencies between attitudes and behaviors concerning diversity and difference within and outside of the organization. In comparison to the previous forms that capture more objective and numeric inconsistencies, this discontinuity accounts for the attitudes toward and approach to diversity that are indicative of an organization's or community's commitment to inclusiveness and valuing of difference. For instance, an employee may live in a community that stigmatizes members of particular identity groups and in which behaviors signal that different social identity groups should not integrate. Conversely, the employee may work in an organization in which diversity is seen as strength and is actively supported and celebrated. Some existing work reflects a concern with perceptions of a community's inclusiveness. For example, Ragins et al (2012) examines perceptions of community diversity climate and its impact on moving intentions; and McKay \& Avery (2006) theorize that the quality of community interactions may play a role in racially diverse applicants' intentions to join an organization. Yet, this work does not directly consider the possible inconsistency between attitudes and behaviors toward diversity experienced within the organization as compared to the within the community. Below, we consider such a case, in which an organizations' efforts to create an environment in which minorities can contribute and flourish is inconsistent with a less inclusive response to diversity and difference in the community.

\section{METHODS}


We engaged in a multi-method qualitative study in which we first conducted a number of focus groups with trainees working at different hospitals located in a city in the Northeast U.S. Then, we partnered with two specific hospitals located in the same city, also within the Northeast U.S. Both hospitals have an established Diversity office, and we were given access to interview hospital leaders (both administrators and faculty) involved with the diversity efforts and trainee development.

The initial goal of our data collection was to gain a general sense of the hospital context, the particular approaches each organization takes to managing diversity, as well a sense of how trainees from underrepresented backgrounds experience their development in these hospitals. To do so, we gathered insight via focus groups with trainees and interviews with diversity leaders. Such an approach allowed for data source triangulation, by generating understanding from both the leadership and employees as it relates to the challenges created by the broader community.

\section{Participants}

Study participants were comprised of two groups of individuals: trainees and hospital leaders. The trainees in our sample $(n=10)$ were medical residents training at various hospitals in the same city. Importantly, they were all from social identity backgrounds that are traditionally underrepresented in medicine ("URM"), which is a categorization used in the field of medicine describing those individuals who are racially or ethnically underrepresented in the medical field. Of the URM trainees, 90\% self-identified as "Black, not of Hispanic origin" and 10\% selfidentified as "Asian or Pacific Islander." The trainee sample was comprised of $40 \%$ males and $60 \%$ females. We recruited these participants by posting fliers in the hospitals, as well as through an email sent out by a local non-profit organization focused on increasing diversity in the professional workforce in that city. 
The hospital leaders in our study $(\mathrm{n}=11)$ were all involved with their organizations' diversity efforts. Some of these leaders were non-physician administrators, and others were faculty/physicians who also hold leadership roles in the diversity office. For the sample of leaders, $82 \%$ self-identified as "Black, not of Hispanic origin" and 18\% self-identified as "Hispanic." Additionally, 55\% of the leaders were male and 45\% female.

\section{Data Collection}

We conducted three trainee focus groups, with 2-4 participants in each group. Focus groups were conducted by a team of 2-3 researchers (authors on this paper), and lasted from 70120 minutes. Focus group questions probed individuals' experiences as minority trainees in their hospitals, the challenges they face in their development, and the support, if any, they received from their organizations. As suggested by Morgan (1998), we attempted to remain flexible with where each group's conversation headed, using follow-up and probing questions where appropriate. At the conclusion of each group, participants completed a small survey capturing relevant demographic information.

Additionally, we conducted semi-structured interviews with leaders in the two specific hospitals to build insight into their formal programs and informal efforts to build an inclusive environment for URM trainees. A team of two researchers (authors on this paper) conducted semi-structured interviews in person with each leader, which lasted approximately 60-90 minutes each. Interview questions explored each leader's role in the hospital and with the diversity office, the challenges they experience to recruiting, retaining, and supporting minority trainees and physicians, and the programming and approaches that each office engages in to support the building of an inclusive culture. All interviews and focus groups were recorded and transcribed verbatim by a professional transcription service to facilitate analysis. 
Throughout data collection, we kept detailed field notes to capture our reflections and commentary on issues and themes that emerged during the interviews and focus groups (Miles \& Huberman, 1994). Our team of researchers met frequently throughout the process to discuss these ongoing reflections and emerging findings.

\section{Data Analysis}

For data analysis, we used an iterative approach built from basic guidelines for qualitative research in Miles and Huberman (1994). Such an approach involves moving iteratively among our data, the literature, and our own emergent ideas in order to elicit common themes that allowed for a deeper, thematic understanding of the organization and community intersection. Through our field notes and team meetings, it became clear that we had a strong story in our data, from both trainees and leaders, concerning discrepancies in experiences of inclusion in the organization and the broader community. At this stage, we revisited existing literature and found some evidence of community considerations in organizational research on diversity, with many open questions concerning how organizations and individual employees experience and make sense of this intersection. Therefore, we organized existing literature to develop our initial framework of inclusion discontinuities, and then entered our systematic coding of the data with these themes and questions in mind.

From this stage, we moved into a systematic process of coding the transcribed interview and focus group data to understand how community issues are integrated into the organizations' inclusion efforts, and how individual trainees discuss their community based experiences in relation to their work lives. In doing so, we followed three steps adapted from a broader method commonly used in inductive qualitative research (e.g., Gioia, Corley \& Hamilton, 2013). First, we engaged in open coding, looking for instances in which leaders or trainees mentioned the 
local community in which their organization is situated. We then pared down these instances to capture only instances when the community was mentioned as relevant in some way to diversity and inclusion. Then, we moved onto the next level of coding, which compared across data fragments from the first round, looking for similarities and differences that clarified if/how leaders and trainees made sense of the community as a challenge to inclusion. In so doing, we moved to "experience-distant" coding, which is more conceptual in nature than in the first round of open coding (Locke, 2001) and allowed us to develop a sense of common themes. Finally, we looked for relationships among the conceptual themes, which allowed us to distinguish themes speaking to challenges or problems created by the community intersection (for leaders and for trainees) from themes that spoke to how the organizations attempted to deal with these challenges.

\section{THE CASE OF MEDICINE: DISCONTINUITY WITH COMMUNITY AS A CHALLENGE TO ORGANIZATIONAL INCLUSION IN HOSPITALS}

"I think we have several pros and several cons [in attracting and retaining diverse employees]. The pros are certainly, you know I mentioned, our CEO who supports these issues not just with word but with deed and resources...I think we're very fortunate in that regard. We're very fortunate to have this Diversity office; we're very fortunate to have the resources, we're very fortunate to have a CEO who cares. I think those are the pros. Then I think there are cons; some things we can't control. OldTown ${ }^{1}$. That always seems unfavorable."

\section{- Hospital Leader}

The story that emerged from our data collection revealed how the local community in which an organization is situated can challenge an organization's internal efforts to foster an inclusive culture. As illustrated by the above quote from one organizational leader, even with important internal levers functioning (e.g., leadership support, a dedicated diversity office, etc.),

\footnotetext{
${ }^{1}$ Pseudonym for the city’s name
} 
the city in which the organization is situated creates an intractable problem that is difficult for the hospital to address. Collectively, our findings in this section demonstrate how discontinuity between the organizations efforts to create an inclusive environment and the community's response to diversity and difference creates challenges for leaders in retaining and supporting minority individuals and for trainees in feeling like they could build a life within, and outside of, their organizations. More specifically, the themes reveal how leaders and trainees make sense of and experience the discontinuity in attitudes toward and approaches to diversity, with historical perceptions, cross-boundary interactions, and cultural and relational concerns driving the complex nature of this challenge. In turn, such forces constrain the extent to which the organization can truly foster an inclusive environment in which minority individuals desire to stay over the longer term.

\section{Historical Perceptions of the Community}

When asked what challenges they face in their diversity efforts, leaders consistently mentioned the surrounding city as a major struggle for them. In elaborating further, they discussed the deep-rooted and historical perceptions of the community as a driver of this struggle. One leader explained:

"OldTown is a huge challenge for us, because people don't want to come here. OldTown is a huge detractor for us in the work that we do in the diversity office. Most people are dying to come to OldTown. Most. But not URMs... We call it more of a myth than a reality, but the OldTown's kind of history. It's not such a diversity-friendly history, with the whole busing and the segregation, etcetera. OldTown does not have a pretty history when it comes to, especially African Americans. So I think that people are a little skittish, to say, especially if they've never been here before."

Historical perceptions of the city often shaped potential trainees' perspectives before they even experienced the community themselves. In the face of such negative perceptions, leaders were challenged in recruiting trainees to their hospital initially and also in convincing individuals to 
stay with the hospital after their training is completed (which is often a goal in top teaching hospitals like the ones we studied, particularly for URM trainees). Therefore, leaders had to convince trainees not only that an inclusive culture exists within the hospital, but also that these individuals could stay with the hospital and build a life in the surrounding community. The following quote from one leader aptly reflects this struggle and its foundation in historical perceptions:

"The biggest challenge is OldTown. No one wants to stay in OldTown unless they were born here or their family lives here now. And we don't quite understand why. I think some of it is historic....I've heard applicants use the words 'segregated city.' And sometimes you can convince applicants that that's not going to matter during residency. So come for three years and get the best training of your life. And they'll buy that. But it's really hard to convince people, 'Come stay here for the rest of your life and start your career here,' if they already think that way."

Another leader similarly echoes this struggle:

"So I think when we approach recruiting, we think about getting them to apply in the first place, which is a big step, because a lot of people don't even want to look at OldTown, just because what they have heard about the history here... And then if you finally get that small percentage of people to come and train for a while, it's convincing them they can stay here for the rest of their lives. If there aren't family draws or cultural draws, it's kind of hard to do that."

Clearly, these leaders have come to understand the complexity created by historical perceptions of the city as an influence on individuals' decisions to join and stay with their hospital over the long term. However, leaders also appear to recognize that their hospitals, as open systems, interact with this the community in ways that extend far beyond what happens when residents leave the hospital at the end of the day. Rather, leaders also recognize that the community transcends the walls of the hospital because community members interact with minority trainees when they come to the hospital as patients or family and friends of patients.

\section{Cross-boundary Interactions between the Hospital and the Community}


Beyond the community's problematic history, patients' and their family members'

negative attitudes towards minority residents became reflective of how the broader community

may respond in diverse interactions. One leader explains how this plays out:

"I think on the front lines there have been some issues... where patients haven't felt comfortable being cared for by minority residents... We've heard several times here: a black [resident] walked into a room and the patient thinking that they're coming to take their food tray...that takes a toll. There might be, throughout the course of your training, some micro incidents that happen, that might impact you. You may feel more socially isolated because you're not in a city like New York where there's more diversity. I think OldTown is diverse, but OldTown's diversity is very much drawn along SES lines; the amount of minority professionals I think is still pretty thin."

In such interactions, patients become 'representative community members' signaling to minority trainees the attitudes and behaviors that characterize the community more broadly. This experience is echoed as one trainee participant recalled a particular negative interaction he had with an elderly patient and his wife:

"[I remember] this super, super, super sick guy.... and his wife.... When she met me, she was like, 'Oh my god, you're black and you work here?' She's like, 'Aren't there other cities in America that you'd apply better? Why would you come up here?' ... And I had one of those out-of-body experiences because I'm like your husband is like dying right here. All I want to do is help with this and yet you're so worried and concerned about whether or not I'm having a good experience in this city."

Such interactions signaled to the trainees how the community responds to and approaches diversity more generally. This spillover effect, in which community members bring representative attitudes and behaviors into the organization, is echoed by Brief and colleagues (2013) in their discussion of the "attitudinal baggage" (negative racial attitudes) that employees bring with them into their workplaces. Yet here, the baggage was not brought in by employees, but rather entered via patients, who were the very individuals that the doctors must treat in their work. Such cross-boundary interactions created another complex layer to the story, particularly 
as the hospital is focused on patient-centered care, but also seeks to create an inclusive culture for trainees and employees.

\section{Cultural \& Relational Considerations: Can I Make a Life Here?}

Importantly, trainees confirmed that community considerations are not only drivers of their decision to join an organization in first place, but also in their decision to stay with the organization over the longer term. In discussing their experiences as URM trainees, many reflected on the extent to which they felt they could build a social and cultural life outside of the organization's walls. Often times, such considerations were discussed as central to their views of their organizational lives, as well as to their career choices going forward. As one participant describes:

"I'm actually at a crossroads right now [as I think about my next career steps.] So that was a big question, you know, would I stay in the area. I wouldn't say it was specific to the [hospital name] because, to be honest with you, I think that my institution does a pretty good job in comparison to all the places that I interviewed at or places that my friends are at in terms of diversity and trying to be sensitive about those issues.... And so it's mostly more of an OldTown thing that really has me thinking very hard about where I'm going to go...."

This same participant elaborated further to discuss how cultural aspects of the community are

less reflective of her background, a key theme echoed by many other trainees as well:

“... in terms of the professional scene and things to do...like finding a place that can play jazz music, for instance, you can't find that. That's popular in African American culture....Most of the places like nightclubs and lounges are predominantly Caucasian, they play top 40 predominantly, you know, music that caters to a more Caucasian population. And so do the concerts as well. You don't see too many R\&B people coming to perform in OldTown. The entertainment is all targeted towards the population in OldTown."

Experiencing this segregation in the city's entertainment became indicative of the community's

broader commitment to inclusiveness of diverse individuals. Another participant, who was 
interviewed just days after she made the decision to leave the organization, explains her choice to us, which similarly echoes this theme:

“... you know, [my husband and I] can't say we love the city. We can't say that we love the cultural aspects of the city. We feel like that's lacking a little bit more than what we had growing up. We do sometimes feel that like all the brown people live in one section, and all the Hispanic people live in one section, and that's a little bit foreign to how we grew up as well... But, there is not a hospital piece. And that's always been the biggest problem is that people always ask me, 'in your ideal world, what would you do?' And I would say, 'I'd move this [hospital name] to [another city].' You know?'

The latter part of this quote powerfully illustrates that this participant's choice to leave was not driven by inclusion in the organization, but rather experiences in the community around it.

In addition to these perceptions of city culture, individuals also expressed concerns about their ability build a social network of friends and relationships in their personal lives. As echoed by one of the leaders:

"I've interviewed people who I would identify as Black, Latino or Native American. I think the biggest non-professional question that I am asked comes from - yes, nonprofessional in the sense of not specifically career-related - is by Black women who are really asking, how can you survive on the social scene in OldTown? That's one of their concerns, as they approach considering working here for anywhere from two to three, or more, years....like women being concerned, am I going to have a social life here? Am I going to be able to date here? Just knowing how difficult OldTown can be for that kind of thing."

This concern illustrates the intertwined connection between career choices and perceptions of the community's social fit for the individual. Another participant's reflection on her future career plans beyond her training hints at the connection between the power and status discontinuity described above and her problem fitting in socially. That is, while there may be racial diversity in the city at large, it is rare for her to find other Black professionals to socialize with. She explains:

"I think even the black professionals that we know, we feel like we know all of them. You know, wherever you go, the same kind of 50 to 100 people. So I think for me...I 
would definitely not pick OldTown after my training because it's not a city where I feel I can totally set up a social network easily and can totally feel comfortable and feel at home right away. ...even in a place like Atlanta, for example, or DC, New York, all those kinds of places, I feel like ....as a Black professional, I could go there, very easily find a community, very, very easily integrate into that city. But in OldTown, I would probably be very, very lonely."

These findings reveal how retention of diverse employees depends in part on whether they perceive that they will feel comfortable, included, and perhaps even find a partner, in the community outside the organization. That is, in discussing how they experience inclusion in the organization, many participants inevitably discussed the inclusiveness experienced in their lives outside of work, in cultural and relational terms.

Together, these themes reveal the complex set of issues that combine to create inconsistencies between the perceived attitudes and actions toward fostering inclusion within the organization and within the community. Driven by historical perceptions, cross-boundary interactions between the hospital and the community, and individuals' perceived social and cultural fit, such inconsistencies impact the organization's ability to foster an inclusive culture in which minority employees want to join and stay. Now, drawing from our case, we consider how organizations can build their capacity to address this difficult challenge.

\section{BUILDING ORGANIZATIONAL CAPACITY TO ADDRESS THIS CHALLENGE}

While challenges to fostering inclusive organizations are never easily overcome, it is even harder to address a challenge that is deeply-rooted in historical perceptions of and relational experiences in the community. There is certainly no perfect answer to this imperfect challenge; yet, organizations like the ones we studied, can build their capacity to work more effectively across difference within and outside of their own walls. At a foundation, "systems thinking" must be mobilized throughout the organization in order to acknowledge, appreciate and discuss 
the influence the broader community has on the organization's inclusion efforts as well as on employees' experiences. The hospital leaders in our study clearly acknowledged the powerful influence the community can have. Until an organization recognizes that building an inclusive culture requires more than just an individual-level focus, or even an organizational-level focus, it will be difficult to address the challenges created by its intersection with the broader community. Mobilizing systems thinking - appreciating and recognizing that community embeddedness matters to diversity efforts - allows the organization to move into implementation mode: that is, to consider, what capacities can we build to address this problem created by the open system in which we operate? We explore next three such capacities, building upon data from the hospitals we studied that were working on addressing this challenge.

\section{Build Capacity for Coalition Building}

From a foundation of systems thinking, organizations can focus on building their capacity for coalition building with other organizations within the community. Coalition building can take two forms: (1) partnering with a local organization that convenes minority professionals from across the city and (2) partnering with other similar organizations in community-based efforts.

One of the hospitals we studied engaged with a non-profit organization that focused specifically on developing minority professionals as leaders in their organizations in this city. The hospital was able to sponsor their minority employees' participation in the non-profit's leadership training programs. In so doing, individual employees were able to build connections with and garner support from other minority professionals in the city, creating a network of support outside of their organizations. On their website, this particular non-profit organization writes: "At a time when our region is losing professionals of color, three-quarters of our program 
graduates credit [our organization] with influencing their decision to stay in OldTown" (organization's website). Thus, partnering with an external organization that reaches a wider array of minority professionals in the community can help to build capacity at both the organizational- and individual-level, and have tangible results for retention. Of course, as with any approach, coalition building in this form can have potential drawbacks. For example, in engaging with an external party that provides such support, an organization must take care not to fully relinquish the responsibility for managing the community-based challenges that affect it. Thus, the hospital we studied partnered with this external organization while also pursuing other strategies.

In addition to engaging with external organizations, coalition building can also take place among organizations themselves. In the case of the hospitals we studied, leaders in the different diversity offices had informal discussions on the common community-based challenges they face. While initial efforts were made to build coalitions between these organizations, there was considerable room for improvement. Recall organizational theory not only suggests that environments are important influences on organizations, but also that organizations have the power to shape environments (Freeman, 1984; Mitroff, 1983). While long-standing community perceptions and relations are certainly not changed easily, organizations working together arguably have more power to shift the communities when resources are invested collaboratively. As Brief, Butz \& Deitch (2013) states, "If we are right about the importance of environments, organizational leaders need to recognize that the resources they expend on improving race relations at work should be coupled with like expenditures within the communities where their employees live." Building a capacity for coalition building with other organizations in the community is an important piece of the process. 


\section{Build Capacity for Open and Honest Dialogue}

In addition to the external focus of coalition building, organizations must also focus internally on building a capacity for real and honest dialogue about the challenges created by the community. In our cases, this took two forms: real and open dialogue in the recruitment process, and discussing head-on issues that arise from cross-boundary interactions with patients.

One primary issue we heard organizational leaders struggling with was how to attend to the questions they get from diverse applicants about the culture and perception of the city. One leader, who is African American himself, discussed his approach with potential employees when they mention their concerns about the city's history of racism:

"when I hear 'OldTown has a history of racism; this is not like comfortable for me...' ...Or really any other Southerner who might say 'Well I hear they are racist.' ... So having that conversation that those are things that concern them. You know of course I'll tell them if I can make it up here if I can adjust then I'm sure you can. But I think that's a key thing: me engaging them and I think when I go to these recruitment conferences and I look at [organizations doing recruiting], I see the successful ones engage the students better, so they are able to identify with them."

This approach echoes a simple sense of honest and open dialogue, as well as identification with a successful leader who is similar to them and has found a way to be comfortable in the community.

Beyond recruitment, real dialogue extends into patient interactions and the attitudinal baggage they may bring with them into the hospital. The organization has two choices: ignore it, or openly acknowledge and do something about it. On the one hand, hospitals are patientcentered organizations and their mission is to serve patients; yet, focusing solely on patient care, at the expense of examining and openly attending to issues that disrupt inclusion, becomes problematic. 
For example, a leader in one of the organizations spoke to us about procedures they put in place to address a patient's explicit request not to be treated by a minority physician. She discussed the difficult conversations that had to take place, particularly in an organization so focused on patient-care ("Our Medical Policy Committee did not allow it to become a policy, because it can't be a policy on how our patients behave. We can't have policy; but, we can have procedures on how to respond.") So, they did just that: a leader from the diversity office worked with each department to create procedures that worked within their context. While every interaction and situation has unique properties, the procedures offer guidelines on what steps to take to respond to this specific, yet fairly common, incident in which a patient requests not to be treated by a minority doctor. The leader explained the discussions she had in this process:

" [We told the departments] these are the guidelines that everybody who is taking care of patients needs to know about, if a patient asks for another physician because of their race, sex, religion, etc.... I explained to [department leaders] how it was very important to first of all, agree what your procedure was going to be within your own department; and then, to make sure that everybody was informed of it."

Importantly, these procedures were communicated to all individuals, including doctors, nurses

and other staff. This illustrates that an important element in fostering real dialogue and treatment of these issues is that it must reach beyond minority employees to majority group members as well. Individuals throughout all levels of the organization and from all backgrounds must be part of the open and honest dialogue about these community intersections. From these more specific examples, we highlight how simply approaching these issues openly moves closer to creating an inclusive experience for employees at the intersection of their organizations and communities.

\section{Build Capacity for Buffering: Creating a Community Within}

Finally, our analysis revealed the great importance of building a 'community within' the organization in which employees can find and build social and support networks that extend 
outside of the organization into their personal lives. In our data, many participants referred to the importance of finding their community within the organization, with fellow residents and doctors who they felt were more "like me" than anyone they could find in the community. As one participant explains:

"As residents it kind of creates community automatically, and it's like all people you work with, get to know very well, they're all very accepting, so that's great; but if it weren't for them, I don't know what I would do with my evenings."

We heard similar reflections from many participants, who discussed how in a community where they feel out of place culturally and relationally, their work colleagues have become an important, and often the only, social network outside of the hospital as well. Given this, we suggest the organization facilitate this internal community building that extends beyond the organization. In the organizations we studied, they created avenues - formal and informal - for employees to support and be social with one another both in and outside of the organization. One participant discussed how an informal social event that was initially created for minority employees extended out to everyone and provided a community for minority and majority employees to come together:

"And [the majority individuals] were like, "It's actually kind of great that you guys have this thing called Social Thursdays the last Thursday in every month, you know, because that just sounds like fun. It's like free bar food and drinks and everyone hanging around. I thought that was huge during residency. Sometimes you just wanted to go somewhere and hang out." So I think that makes it - it's kind of like having another family within to identify with."

While formalized affinity groups and networking events designed for underrepresented employees are a useful start, this example echoes the importance of creating the space for community to form among majority and minority employees, in a way that fulfills their identity and relational needs in more of a social sense.

CONTRIBUTIONS \& FUTURE RESEARCH 
In this paper, we conceptualize and empirically illustrate how inconsistencies between an organization's and community's attitudes toward and responses to diversity and difference create a complex, and less attended to, challenge for organizational diversity management efforts. We also illustrate what organizations can do to help build capacity to address these concerns. In doing so, we make several contributions of interest to both scholars and organizations.

First, we introduce the concept of inclusion discontinuity as an inconsistency between an organization's inclusiveness and that in its community. To be sure, existing work has considered particular community measures (some demographic, some power/status, some attitudes) that impact diversity relevant outcomes in organization. In our paper, we integrate this small body of work into a broader framework of inclusion discontinuities, which shows that the challenge to organizational inclusion may actually be driven by the inconsistencies. In other words, beyond the direct impact of community variables on organizational outcomes, the actual gap between the organization and its community on diversity relevant dimensions may have important impacts as well. Secondly, through our study in the context of medicine, we offer insight into how organizations may perceive and experience their intersection with the community as relevant to their inclusion efforts. Specifically, we illustrate how historical perceptions, cross-boundary interactions, and employees' cultural and relational concerns combine to create a complex and deep-rooted challenge. Finally, through our case study of organizations grappling with inclusion discontinuity with the community, we offer insight into how organizations can build their capacity to address this difficult challenge.

The foundation provided here opens doors for future research to deepen our understanding of inclusion discontinuities with the community and their impact on organizational inclusion efforts. First, future research could examine how the magnitude of the 
inconsistency may matter. For example, is there a tipping point at which a larger perceived inconsistency creates a stronger impact on recruitment or retention of members from underrepresented groups? Further, future research could also build insight into the interactions among particular forms of inclusion discontinuity. For example, do more objective discontinuities (e.g., demographic) interact with more subjective measure (e.g., in attitudes and behaviors), resulting in a relatively stronger effect on recruitment and retention? Alternatively, in the face of objective inconsistencies in demographic representation and power and status, can organizations and communities work to lessen the perceived disconnect in attitudes and behaviors? These complex questions are crucial to deepening our understanding of the organization and community intersection.

In this paper, we focused on discontinuities in one direction: when an organization puts forth effort to create inclusion, yet is situated in a community that is less inclusive. In the future, research might consider a comparative case - examining the similarities and differences in individuals' experiences of the opposite direction: that is when communities in which inclusion is high, and the organizational experience is less inclusive. To be sure, much research focuses on building more inclusion within organizations; but, there's room to consider this in conjunction with the community. Perhaps spillover can occur in a positive direction, in which an organization can learn and build from a foundation of inclusion demonstrated in the community.

Finally, in our paper, we offer areas in which organizations can build their capacity to address the complex challenge created by inclusion discontinuity with the community. In the future, it may be possible to conduct an interventional study, in which outcomes are captured longitudinally within an organization to see if/how such efforts can create even small shifts in perceptions of and impacts derived from inconsistencies with the community. Additionally, a 
comparative study across multiple communities could offer promise in understanding how inclusive cultures in organizations may be built in communities that are more aligned in terms of their approach to and valuing of diversity and difference.

In sum, in this paper we shed light on the impacts of inclusion discontinuities on an organization's ability to build an inclusive organizational culture and we demonstrate several ways that organizations can address these concerns. Our study demonstrates that it is not enough for organizations to focus internally on working across difference; rather recognizing that employees also interact with communities with a history, attitudes, and practices toward diversity is vital for fostering inclusion both within and outside of their own walls. 


\section{References}

Ainscow, M., and Sandill, A. (2010), "Developing inclusive education systems: the role of organisational cultures and leadership", International Journal of Inclusive Education, Vol. 14 No. 4, pp. 401-416.

Avery, D.R., McKay, P.F., and Wilson, D.C. (2008), "What are the odds? How demographic similarity affects the prevalence of perceived employment discrimination", Journal of Applied Psychology, Vol. 93 No. 2, pp. 235-249.

Barnard, C.I. (1938), The Functions of the Executive, Harvard University Press, Cambridge, MA.

Brief, A.P., Butz, R.M., and Deitch, E.A. (2013), "Organizations as reflections of their environments: The case of race composition", in R.L. Dipboye \& A. Collella (Eds.), The Psychological Bases of Discrimination at Work, Jossey-Bass, San Francisco, CA.

Brief, A.P., Umphress, E.E., Dietz, J., Burrows, J.W., Butz, R.M., and Scholten, L. (2005), "Community matters: Realistic group conflict theory and the impact of diversity", Academy of Management Journal, Vol. 48 No. 5, pp. 830-844.

Cox, T. (1994), Cultural diversity in organizations: Theory, research and practice, BerrettKoehler, San Francisco, CA.

Davidson, M. (2011), The End of Diversity as We Know It: Why Diversity Efforts Fail and How Leveraging Difference Can Succeed, Berrett-Koehler, San Francisco, CA.

Ely, R.J. and Thomas, D.A. (2001), "Cultural Diversity at Work: the Effects of Diversity Perspectives on Work Group Processes and Outcomes", Administrative Science Quarterly, Vol. 46 No. 2, pp. 229-273.

Freeman, R.E. (1984), Strategic Management: A Stakeholder Approach, Pitman, Boston, MA.

Garnett, F.G. (2012), "Community matters: Uncovering the societal mechanisms undergirding workplace discrimination and inequality", dissertation, University of Michigan, Ann Arbor.

Gersick, C. (1988), "Time and transition in work teams: Toward a new model of group development", Academy of Management Journal, Vol. 31 No. 1, pp. 9-41.

Gioia D. A., Corley, K. G. and Hamilton, A. L. (2013), "Seeking Qualitative Rigor in Inductive Research: Notes on the Gioia Methodology", Organizational Research Methods, Vol. 16 No.1, pp 15-31.

Gordon, D. M., Edwards, R. and Reich, M. (1982), Segmented work, divided workers: The historical transformation of labor in the United States, Cambridge University Press, New York. 
Joshi, A., and Roh, H. (2009), "The role of context in work team diversity research: a metaanalytic review", Academy of Management Journal, Vol. 52 No. 3, pp. 599-627.

Katz, D., and Kahn, R.L. (1978), The Social Psychology of Organizations, (2 ${ }^{\text {nd }}$ Ed.), Wiley, New York.

King, E.B., Dawson, J.F., West, M.A., Gilrane, V.L., Peddie, C.I., and Bastin, L. (2011), "Why organizational and community diversity matter: Representativeness and the emergence of incivility and organizational performance", Academy of Management Journal, Vol. 54 No. 6, pp. 1103-1118.

Lenski, G.E. (1966). Power and privilege: A theory of social stratification, UNC Press.

Leonard, J. S., Levine, D. I., \& Joshi, A. (2004), "Do birds of a feather shop together? The effects on performance of employees' similarity with one another and with customers", Journal of Organizational Behavior, 25: pp. 731-754.

Locke, K. (2001), Grounded Theory in Management Research, Thousand Oaks: Sage.

Marquis, C., Lounsbury, M., and Greenwood, R. (2011), "Introduction: Community as an Institutional Order and a Type of Organizing", in Marquis, C., Lounsbury, M., and Greenwood, R. (Ed.), Communities and Organizations (Research in the Sociology of Organizations Volume 33), Emerald Group Publishing Limited, pp. ix-xxvii

McKay, P.F. and Avery, D.R. (2006), "What has race got to do with it? Unraveling the role of racioethnicity in job seekers' reactions to site visits", Personnel Psychology, Vol. 59, pp. 395-429.

Merriam-Webster. (2013), "Discontinuity”, available at: http://www.merriamwebster.com/dictionary/discontinuity (accessed 18 November 2013).

Miles, M. B., and Huberman, A. M. (1994), Qualitative Data Analysis: An Expanded Sourcebook, (2 ${ }^{\text {nd }}$ Ed.), Sage, Thousand Oaks, CA.

Mitroff, I.I. (1983), Stakeholders of the organizational mind, Jossey-Bass, San Francisco, CA.

Moore, C., Wisnivesky, J., Williams, S. and McGinn T. (2003), "Medical errors related to discontinuity of care from an inpatient to an outpatient setting", Journal of General Internal Medicine, Vol. 18 No. 8, pp. 646-51.

Morgan, D. L. (1988), Focus groups as qualitative research, Sage, Newbury Park, CA.

Nishii, L.H., and Mayer, D.M. (2009), "Do inclusive leaders help to reduce turnover in diverse groups? The moderating role of leader-member exchange in the diversity to turnover relationship", Journal of Applied Psychology, Vol. 94 No. 6, pp. 1412. 
Pless, N.M. and Maak, T. (2004), "Building an inclusive diversity culture: Principles, processes and practice", Journal of Business Ethics, Vol. 54, pp. 129-147.

Pugh, S.D., Dietz, J., Brief, A.P., and Wiley, J.W. (2008), "Looking inside and out: The impact of employee and community demographic composition on organizational diversity climate", Journal of Applied Psychology, Vol. 93 No. 6, pp. 1422-1428.

Ragins, B.R., Gonzalez, J.A., Ehrhardt, K., and Singh, R. (2012), "Crossing the threshold: The spillover of community racial diversity and diversity climate to the workplace", Personnel Psychology, Vol. 65, pp. 755-787.

Sacco, J. M. and Schmitt, N. (2005), "A dynamic multilevel model of demographic diversity and misfit effects," Journal of Applied Psychology, Vol. 90, No. 2: pp. 201-231.

Selznick, P. (1949), TVA and the Grass Roots: a Study in the Sociology of Formal Organization, University of California Press, Berkeley.

Thomas, R.R. (1991), Beyond Race and Gender: Unleashing the Power of Your Total Workforce by Managing Diversity, Amacon, NY. 